\title{
ESTADO NUTRICIONAL DE PACIENTES DIABÉTICOS ATENDIDOS EM UMA UNIDADE BÁSICA DE SAUDE, CAMPINAS-SP*
}

\author{
Michele Tatiane Lagacci ${ }^{1}$, Tâmara Ligia Verussa ${ }^{1}$, Vanessa Grazielle Vieira ${ }^{1}$, Mirian Ueno², Monize Cocetti ${ }^{3}$, Maria \\ Filomena Ceolim ${ }^{4}$, Maria Helena de Melo Lima ${ }^{4}$
}

\begin{abstract}
RESUMO: O estudo objetivou avaliar a composição corporal e o perfil bioquímico de indivíduos diabéticos. Participaram 105 pacientes, foram realizadas medidas antropométricas, composição corporal por bioimpedância elétrica e exames bioquímicos, sendo que $70 \%$ relataram apresentar a doença há mais de 5 anos e $43,3 \%$ eram obesos (IMC=30). As mulheres apresentaram maior IMC $(30,65 \pm 4,45 \mathrm{Kg} / \mathrm{m} 2$ vs $28,04 \pm 5,21 \mathrm{Kg} / \mathrm{m} 2)$ e percentual de gordura corporal $(38,19 \pm 4,49 \%$ vs

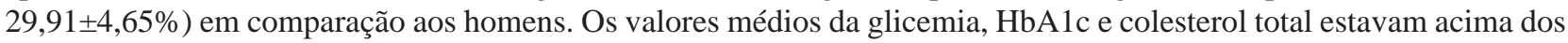
valores de referência, em ambos os sexos. Estes resultados indicam a necessidade de capacitar e implementar intervenções no âmbito da prevenção secundária nesta população, com o objetivo de reduzir as complicações e problemas decorrentes do diabetes, com vistas a melhoria de sua qualidade de vida.
\end{abstract}

PALAVRAS-CHAVE: Estado nutricional; Diabetes Mellitus.

\section{NUTRITIONAL STATUS OF DIABETIC PATIENTS ASSISTED AT A PRIMARY CARE UNIT IN THE CITY OF CAMPINAS/SAO PAULO-BRAZIL}

\begin{abstract}
The study objectifies to evaluate body composition and biochemical profile of diabetic subjects. 105 patients participated, anthropometric measures, evaluation of body composition by bioelectrical impedance and biochemical tests were carried out. $70 \%$ of the patients reported being diagnosed at least 5 years ago; $43.3 \%$ were obese $(\mathrm{BMI}=30)$ and women presented higher values for BMI $(30.65 \pm 4.45 \mathrm{Kg} / \mathrm{m} 2$ vs $28.04 \pm 5.21 \mathrm{Kg} / \mathrm{m} 2)$ and percentual body fat $(38.19 \pm 4.49 \%$ vs $29.91 \pm 4.65 \%)$ than men. Average values of glycemia, HbA1c and total cholesterol were above reference values for both sexes. Results suggest the need for qualification and intervention development in the secondary prevention scope in order to reduce complications and problems related to diabetes, thus improving quality of life for those patients.
\end{abstract}

KEYWORDS: Nutritional status; Diabetes Mellitus.

\section{ESTADO NUTRICIONAL EN PACIENTES DIABÉTICOS ATENDIDOS EN UNA UNIDAD BÁSICADE SALUD, CAMPINAS/SAO PAULO-BRASIL}

RESUMEN: El objetivo de este estudio fue evaluar la composición corporal y perfil bioquímico de individuos diabéticos. Participaron 105 pacientes y fueron realizadas medidas antropométricas, composición corporal por bioimpedancia eléctrica y exámenes bioquímicos. 70\% describieron presentar la enfermedad a mas de cinco años y 43,3\% eran obesos (IMC=30). Las mujeres presentaron mayor IMC ( $30,65 \pm 4,45 \mathrm{Kg} / \mathrm{m} 2$ vs $28,04 \pm 5,21 \mathrm{Kg} / \mathrm{m} 2)$ y percentual de grasa corporal $(38,19 \pm 4,49 \%$ vs $29,91 \pm 4,65 \%$ ) en comparación a los hombres. Los valores medios de glicemia, HbA1c y colesterol total estaban por arriba de los valores de referencia, en ambos sexos. Estos resultados indican la necesidad de implementar intervenciones en el ambito de la prevención secundaria en esta población, a fin de reducir las complicaciones y problemas decorrentes de la diabetes, con la intención de mejorar su calidad de vida.

PALABRAS CLAVE: Estado nutricional; Diabetes Mellitus.

\footnotetext{
*Este trabalho foi financiado pela Fundação de Amparo a Pesquisa do Estado de São Paulo (Fapesp). Processo no 04/10513-6

${ }^{1}$ Acadêmicas. Bolsistas de Iniciação Científica. Curso de Graduação em Enfermagem. Faculdade de Ciências Médicas da Universidade Estadual de Campinas-UNICAMP.

${ }^{2}$ Nutricionista. Pesquisadora do Departamento de Clínica Médica. Faculdade de Ciências Médicas da UNICAMP.

${ }^{3}$ Nutricionista. Pesquisadora no Centro de Investigação em Pediatria-Ciped. Faculdade de Ciências Médicas da UNICAMP.

${ }^{4}$ Doutora. Professora do Departamento de Enfermagem da Faculdade de Ciências Médicas da UNICAMP.
}

Autor correspondente:

Maria Helena de Melo Lima

Rua Tessália Viera de Camargo, 126 - 13098-301 - Campinas-SP.

E-mail:melolima@fcm.unicamp.br 


\section{INTRODUÇÃO}

O diabetes mellitus (DM) atinge em todo o mundo grande número de pessoas de qualquer condição social. Há estimativa de que em 2000 havia 171 milhões de portadores do agravo no mundo e projeção de 366 milhões no ano de 2030. A importância do DM nas últimas décadas vem crescendo em decorrência de vários fatores como, a industrialização, o sedentarismo, a obesidade, o aumento da expectativa de vida e maior sobrevida dos diabéticos ${ }^{(1)}$.

A prevalência do DM 2 é de três a sete vezes maior em obesos do que em indivíduos de peso adequado. Pessoas com Índice da Massa Corpórea (IMC) $>35 \mathrm{Kg} / \mathrm{m}^{2}$ são 20 vezes mais propensos a desenvolver diabetes do que indivíduos com IMC entre 18,5 e $24,9 \mathrm{~kg} / \mathrm{m}^{2(2)}$.

Os portadores de diabetes necessitam modificar hábitos alimentares e aderir a esquemas terapêuticos restritivos, como aplicação diária de insulina e monitorização glicêmica. Também, devem lidar com o fato de ter que conviver por toda vida com a doença que é responsável por complicações clínicas que prejudicam a sua saúde. Todas estas representam, seguramente, um agravo que produz significativo impacto na qualidade de vida de seus portadores.

Há evidências de que mudanças de estilo de vida possam ocorrer com maior sucesso quanto mais precoces forem as intervenções no processo saúdedoença. Também não há controvérsias de que a adoção de uma alimentação saudável, rica em frutas, verduras, legumes, grãos integrais e pobre em gorduras saturadas associada à prática freqüente de atividades físicas, ao menos trinta minutos ao dia, possam atuar beneficamente na qualidade de vida da população e, conseqüentemente, na incidência de doenças crônicas na população em geral ${ }^{(3)}$.

Considerando a importância do DM como problema de saúde pública, é relevante conhecer o portador de diabetes a fim de identificar subsídios que fundamentem intervenções eficazes dos serviços de saúde na prevenção e controle de danos que a doença pode provocar. Assim, este estudo objetivou avaliar o estado nutricional e perfil bioquímico de indivíduos diabéticos assistidos em uma Unidade Básica de Saúde do município de Campinas, SP.

\section{METODOLOGIA}

Trata-se de estudo transversal desenvolvido em uma Unidade Básica de Saúde do Distrito Leste, no município de Campinas, SP, entre janeiro e junho de 2005. Participaram do estudo, após assinatura de Termo de Consentimento Livre e Esclarecido, 105 usuários, 68 mulheres e 37 homens, constituindo uma amostra de conveniência dentre os freqüentadores do grupo de educação em diabetes em andamento no campo da pesquisa.

Os critérios de inclusão de usuários na população de estudo corresponderam a: concordância em participar do estudo; idade igual ou superior a 18 anos para ambos os sexos; pelo menos um ano de diagnóstico confirmado de DM; pelo menos um mês de participação no Grupo de Educação em Diabetes, e capacidade de estabelecer comunicação verbal em situação de entrevista.

Os usuários foram entrevistados no próprio serviço de saúde ou em seus domicílios. Nessa mesma oportunidade ou em outra previamente agendada, e em local de escolha dos usuários, procedeu-se à verificação de medidas antropométricas e à coleta de sangue para dosagem bioquímica.

As medidas antropométricas corresponderam ao peso, estatura, circunferência da cintura e avaliação da composição corporal pelo método de bioimpedância elétrica (BIA) com uso do equipamento BIODYNAMICS modelo 310. Foram utilizados os procedimentos descritos por Lohman et $\mathrm{al}^{(4)}$ e recomendados pela Organização Mundial da Saúde ${ }^{(5)}$. Para identificação do estado nutricional utilizou-se o IMC, segundo o qual o peso Adequado ou Eutrófico corresponde à faixa de 18,5 a 24,9 kg/ $\mathrm{m}^{2}$, o Sobrepeso à 25 à $29,9 \mathrm{~kg} / \mathrm{m}^{2}$ e a Obesidade ao IMC maior ou igual a $30 \mathrm{~kg} / \mathrm{m}^{2(6)}$. Para as medidas de circunferência da cintura adotou-se a referência preconizada pela Organização Mundial de Saúde $(\mathrm{WHO})^{(7)}$ que considera como risco aumentado os valores $\geq 94 \mathrm{~cm}$ para os homens e $\geq 80 \mathrm{~cm}$ para as mulheres e significativamente aumentado quando $\geq 102 \mathrm{~cm}$ e $\geq 88 \mathrm{~cm}$, respectivamente.

A coleta de sangue respeitou o jejum prévio de 8 a 10 horas. Os exames bioquímicos foram realizados por um laboratório contratado. Utilizaramse como referência para nível glicêmico e de hemoglobina glicada os valores preconizados pela Sociedade Brasileira de Diabetes ${ }^{(8)}$, ou seja, glicemia de jejum normal até $99 \mathrm{mg} / \mathrm{dl}$, e hemoglobina glicada menor que 7\%. Para os lípides séricos, consideraramse ideais os seguintes níveis: colesterol total inferior a $200 \mathrm{mg} / \mathrm{dl}$; HDL superior a $45 \mathrm{mg} / \mathrm{dl}$; LDL inferior 
a $100 \mathrm{mg} / \mathrm{dl}$ e triglicérides inferior a $150 \mathrm{mg} / \mathrm{dl}^{(9)}$.

Para elaboração do banco de dados e análise descritiva, foi utilizado o programa Epi Info 6.02. O teste do Qui-quadrado ou o teste Exato de Fisher foram utilizados para verificar associação entre variáveis categóricas. Para comparação de variáveis numéricas entre dois grupos foi utilizado o teste não-paramétrico de Mann-Whitney, pois os dados não apresentavam distribuição normal ou homogeneidade das variâncias para essas variáveis. Foram consideradas significativas as diferenças com $\mathrm{p}<0,05$.

O presente estudo foi aprovado pelo Comitê de Ética em Pesquisa da Faculdade de Ciências Médicas da Unicamp. Protocolo No 492/2004.

\section{RESULTADOS}

Foram avaliados 105 pacientes, todos portadores de DM 2, 68 do sexo feminino (65,38\%) com média de idade de 61,96 $\pm 11,10$ anos, e 37 do sexo masculino (34,62\%), com a média de idade de $62,13 \pm 9,92$ anos. Entre os entrevistados 63,8\% referiram ser casados e 84,8\% alfabetizados. Em relação ao consumo de tabaco e de álcool, 38,1\% relataram ser fumantes e $21,0 \%$ indicaram uso de bebidas alcoólicas. A ocupação mais freqüentemente citada foi o trabalho doméstico (38,5\%), seguida da condição de aposentados e pensionistas (29,8\%).

Quanto ao tempo de diagnóstico do diabetes, $72,1 \%$ dos pacientes referiram conviver com a doença há mais de 5 anos. As doenças crônicas mais citadas pelos pacientes, concomitantes ao diabetes, foram hipertensão arterial (75,2\%) e dislipidemias (15,2\%).

As características do estado nutricional e composição corporal foram avaliadas conforme o sexo e estão apresentadas na Tabela 1.

Tabela 1 - Média e desvio padrão das características físicas dos 105 sujeitos incluídos no estudo. Campinas, 2005

\begin{tabular}{lccc}
\hline & Feminino & Masculino & \\
& $\mathrm{N}=67$ & $\mathrm{n}=37$ & \\
Variáveis & $\overline{\mathrm{x}} \pm \mathrm{DP}$ & $\overline{\mathrm{x}} \pm \mathrm{DP}$ & 0,030 \\
\hline Peso $(\mathrm{Kg})$ & $72,18 \pm 2,50$ & $80,29 \pm 18,29$ & 0,000 \\
Altura $(\mathrm{cm})$ & $153,42 \pm 7,37$ & $168,72 \pm 6,59$ & 0,005 \\
$\mathrm{IMC}(\mathrm{kg} / \mathrm{m} 2)$ & $30,65 \pm 4,45$ & $28,04 \pm 5,21$ & 0,815 \\
Circunferência da cintura $(\mathrm{cm})$ & $102,03 \pm 11,98$ & $102,87 \pm 13,12$ & 0,000 \\
\% de massa gorda & $38,19 \pm 4,49$ & $29,91 \pm 4,65$ & 0,0079 \\
Massa gorda $(\mathrm{Kg})$ & $27,33 \pm 6,26$ & $25,01 \pm 8,67$ & 0,000 \\
Massa magra $(\mathrm{Kg})$ & $43,74 \pm 6,87$ & $56,01 \pm 10,86$ & \\
\hline
\end{tabular}

* Mann-Whitney

A Tabela 2 demonstra o estado nutricional dos pacientes entrevistados de acordo com o IMC e sexo.

Tabela 2 - Estado nutricional segundo IMC e sexo. Campinas, 2005

\begin{tabular}{cccc}
\hline IMC & $\begin{array}{c}\text { Mulheres* } \\
\mathrm{n}(\%)\end{array}$ & $\begin{array}{c}\text { Homens } \\
\mathrm{n}(\%)\end{array}$ & $\begin{array}{c}\text { Total } \\
\mathrm{n}(\%)\end{array}$ \\
\hline $18,5-24,9$ & $6(9,0)$ & $13(35,1)$ & $19(18,2)$ \\
$25-29,9$ & $27(40,3)$ & $13(35,1)$ & $40(38,5)$ \\
$\geq 30$ & $34(50,7)$ & $11(29,8)$ & $45(43,3)$ \\
\hline
\end{tabular}

*uma paciente não completou a entrevista. $x=11,54$; $\mathrm{p}<0,003$

A média da massa magra, segundo a faixa etária e sexo é apresentada na Tabela 3.
Tabela 3 - Medidas descritivas da massa magra, segundo faixa etária e sexo. Campinas, 2005

\begin{tabular}{lccccc}
\hline $\begin{array}{l}\text { Faixa } \\
\text { etária }\end{array}$ & \multicolumn{3}{c}{ Mulheres } & \multicolumn{3}{c}{ Homens } \\
& $\mathrm{n}$ & $\overline{\mathrm{x}} \pm \mathrm{DP}$ & $\mathrm{N}$ & $\overline{\mathrm{x}} \pm \mathrm{DP}$ & $\mathrm{P}^{*}$ \\
\hline $30-49$ & 5 & $50,56 \pm 6,42$ & 4 & $67,15 \pm 11,89$ & 0,086 \\
$50-59$ & 18 & $47,06 \pm 5,28$ & 10 & $56,94 \pm 11,69$ & 0,014 \\
$60-69$ & 18 & $43,66 \pm 4,23$ & 9 & $55,41 \pm 11,29$ & 0,003 \\
70 e + & 13 & $36,64 \pm 5,96$ & 10 & $51,17 \pm 6,34$ & 0,000 \\
\hline * Mann-Whitney & & & &
\end{tabular}

O perfil bioquímico da população estudada é apresentado na Tabela 4. 
Tabela 4 - Valores dos exames bioquímicos da população estudada. Campinas, 2005

\begin{tabular}{lcccc}
\hline & Mulheres & Homens & \\
Exames bioquímicos & $\mathrm{N}=54$ & $\mathrm{n}=34$ & Valor de referência & - \\
& $\overline{\mathrm{x}} \pm \mathrm{DP}$ & $\overline{\mathrm{x}} \pm \mathrm{DP}$ & & $\mathrm{p}$ \\
\hline Hemoglobina glicosilada \% & $7,64 \pm 2,17 * 1$ & $8,33 \pm 2,67 * 2$ & 4,00 a 7,00 & 0,159 \\
Colesterol total (mg/dl) & $204,14 \pm 41,93$ & $201,82 \pm 58,17$ & $\begin{array}{c}\text { Desejável: até } 200 \\
\text { Limítrofe: } 200 \text { a } 239\end{array}$ & 0,337 \\
& & & Alto: superior a 240 & \\
Glicose (mg/dl) & $169,27 \pm 65,88$ & $173,20 \pm 67,90$ & Normais: 60 a 99 & 0,604 \\
HDL (mg/dl) & $44,31 \pm 12,05$ & $38,73 \pm 8,62$ & Desejável: $>45$ & 0,038 \\
LDL (mg/dl) & $126,06 \pm 40,92 * 3$ & $121,56 \pm 32,36$ & Desejável: $<100$ & 0,534 \\
VLDL (mg/dl) & $32,92 \pm 14,64 * 3$ & $33,14 \pm 15,18$ & Até 40 & 0,941 \\
Triglicérides (mg/dl) & $171,24 \pm 86,18$ & $182,91 \pm 106,04$ & Desejáveis: $<150$ & 0,771 \\
\hline
\end{tabular}

${ }^{* 1} \mathrm{O}$ índice variou entre 4,53 a $14,93 \%$

${ }^{* 2} \mathrm{O}$ índice variou entre 5,75 a 19,00\%

${ }^{*}$ Não foi possível calcular LDL e VLDL em um exame.

**Mann-Whitney

\section{DISCUSSÃO}

A prevalência do DM vem aumentando e não há perspectiva de queda, pelo contrário, espera-se um incremento ainda maior, principalmente em países em desenvolvimento com aumento da freqüência nos mais jovens, em que o impacto sobre a qualidade de vida é maior. O DM acarreta uma grande carga econômica e social pois existe uma alta freqüência de complicações agudas e crônicas que levam a hospitalizações, incapacitações, comprometimento da produtividade e morte prematura.

No grupo estudado o número de mulheres foi quase duas vezes maior do que de homens, o que se aproxima do observado no estudo realizado em Viçosa (MG), onde 78,4\% pertenciam ao sexo feminino ${ }^{(10)}$. A maior concentração de mulheres utilizando os serviços de atenção primária em diabetes, tanto pode sugerir uma preocupação destas com sua própria saúde, quanto uma facilidade de acesso aos serviços.

Verificamos que $40 \%$ dos entrevistados referiram a prática de atividade física regular. O exercício físico tem efeito positivo no controle glicêmico e redução das complicações cardiovasculares em portadores de $\mathrm{DM}^{(11)}$. Estudos de base populacional neste sentido são escassos no Brasil, entretanto, uma pesquisa realizada na cidade do Rio de Janeiro, sugere que o sedentarismo é mais freqüente entre mulheres e indivíduos de baixa escolaridade ${ }^{(12)}$. O Diabetes
Prevention Program Group ${ }^{(13)}$ demonstrou que a prática de atividades físicas associada à dieta saudável, pode levar a redução significativa da incidência dos casos de diabetes, sendo essa medida, muitas vezes, mais efetiva do que o uso das drogas na prevenção primária do diabetes tipo 2 . O controle de fatores de risco passíveis de modificação como peso, hábito alimentar, uso do tabaco e prática de atividades físicas, podem reduzir o risco de indivíduos com antecedente familiar de diabetes ${ }^{(3)}$.

Estudos demonstraram que o controle de peso e o aumento da atividade física diminui a resistência à insulina, reduzindo as chances de desenvolvimento do diabetes mellitus ${ }^{(3)}$. Além disso, estudos desenvolvidos pela UKPDS ${ }^{(14)}$ mostraram que pacientes diabéticos mantidos em condições de controle clínico e metabólico apresentam retardo no aparecimento e/ou na progressão de complicações crônicas.

Os valores médios da circunferência da cintura apresentaram-se semelhantes entre os sexos, entretanto quando observamos esses valores, segundo a classificação $\mathrm{WHO}^{(7)}, 92,53 \%$ das mulheres são classificadas com risco significativamente aumentado, enquanto entre os homens esta proporção foi de 51,35\%.

É bem descrito que o envelhecimento acarreta mudanças na composição corporal, principalmente nas proporções e densidades dos componentes da massa livre de gordura $^{(15)}$. Essa alteração foi observada no presente estudo pela avaliação da composição corporal 
segundo faixa etária e sexo. Houve redução na quantidade de massa magra conforme aumento a idade, tanto em mulheres quanto em homens. Estudos demonstram que entre as idades de 25 e 65 anos há um decréscimo substancial na massa magra (10 a 16\%) devido a perdas de massa óssea, músculo-esquelética e água corporal total ${ }^{(16)}$.

A análise do estado nutricional segundo o IMC e sexo, mostra que a maioria das mulheres apresenta sobrepeso (40,3\%) e obesidade (50,7\%), enquanto a maior parte dos homens encontra-se dentro da faixa normal (35,13\%) e de sobrepeso (35,13\%), fato que indica diferenças significativas entre as categorias do estado nutricional em função do sexo $(p<0,003)$. A presença de sobrepeso e obesidade exerce influência considerável na elevada morbidade e mortalidade da doença decorrente principalmente da associação com a doença cardiovascular ${ }^{(17)}$, que é a principal causa de mortalidade em pacientes com DM2 ${ }^{(18)}$.

Em relação as variáveis bioquímicas investigadas, encontramos valores plasmáticos alterados de glicemia de jejum, hemoglobina glicada, colesterol total, HDL-colesterol e triglicérides. Apesar de os resultados serem similares em ambos os sexos, os níveis de triglicérides apresentaram-se mais elevados no sexo masculino. Estudos anteriores demonstraram correlação positiva entre consumo de gorduras saturadas e os níveis de glicemia, maior risco de progressão de tolerância diminuída à glicose para o diabetes $^{(19)}$ e uma correlação negativa com o consumo de ácidos graxos ômega- $3^{(20)}$.

\section{CONCLUSÃO}

As complicações crônicas estão relacionadas com o grau do controle metabólico, ou seja, pacientes com um bom controle apresentam menor incidência das complicações em relação aqueles mal controlados. O tratamento terapêutico, o acompanhamento nutricional e a educação em saúde têm como objetivo retardar o aparecimento de complicações crônicas e melhorar a qualidade de vida, com possibilidades de redução de co-morbidades e da mortalidade específica.

Estudos adicionais que permitam o conhecimento da realidade local podem subsidiar o planejamento de ações efetivas, bem como servir de base para avaliar o impacto de futuras intervenções. Os resultados deste estudo sugerem que um maior investimento na capacitação das equipes de saúde facilitaria um melhor aproveitamento do potencial do
Programa Saúde da Família para a promoção da educação em grupo de doenças crônicas. A equipe capacitada e ações educativas efetivas favorecem a adaptação à condição crônica de saúde e assim, incentivam os portadores da doença a efetuarem um controle metabólico mais adequado, com a finalidade de reduzir as complicações decorrentes da doença e melhorar a qualidade de vida.

\section{REFERÊNCIAS}

1. Rathmann W, Giani G. Global prevalence of diabetes: estimates for the year 2000 and projections for 2030 . Diabetes Care. 2004;27(5):1047-53

2. Mokdad AH, Ford ES, Bowman BA, Dietz WH, Vinicor F, Bales VS et al. Prevalence of obesity, diabetes, and obesity-related health risk factors. JAMA. 2003;289(1):76-9

3. Tuomilehto J, Lindstm J, Eriksson JG, Valle TT, Hamalainen H, Hanne-Parikka P et al. Prevention of type 2 diabetes mellitus by changes in lifestyle among subjects with impaired glucose tolerance. New Engl J Med. 2001;344(18):1343-50.

4. Lohman TG, Roche AF, Martorell R, editors. Anthropometric standardization reference manual. Champaign, IL: Human Kinetics; 1988. p. 177.

5. World Health Organization (WHO). Physical status: the use and interpretation of anthropometry. Geneva; WHO 1995.

6. Fagundes AA, Barros DC, Duar HA, Sardinha LMV, Pereira MM, Leão MM. Sistema de vigilância alimentar e nutricional: orientações básicas para a coleta, o processamento, a análise de dados e a informação em serviços de saúde. Brasília; 2004.

7. World Health Organization. Obesity: preventing and managing the global epidemic. Report. Geneva; 2000. (WHO-Technical Report Series, 894).

8. Consenso Brasileiro de Diabetes. Valores de glicemia para diagnóstico. São Paulo; 2002.

9. Diretriz Brasileira de Diagnóstico e Tratamento da síndrome metabólica. Arq Bras Cardiol. 2005;84(supl1): 03-28.

10. Batista MCR, FranceschinI SCC, Priore SE. Avaliação de indicadores antropométricos de adultos e idosos brasileiros. Nutrire Rev Soc Bras Alim Nutr. 2002;23:67-78 
11. Lindstron J, Louheranta A, Mannelin M, Rastas M, Salminen V, Eriksson J, et al. The Finnish Diabets prevention Study (DPS): lifestyle intervention and 3year results on diet and physical activity. Diabetes Care. 2003;26:3230-6

12. Gomes VB, Siqueira KS, Sichieri R. atividade física em uma amostra probabilística da população do Município do Rio de Janeiro. Cad Saúde Públ. 2001;17:969-76.

13. Diabetes Prevention Program Group. Reduction in the incidence of type 2 diabetes with lifestyle intervention or metformin. N Engl J Med. 2002;346 (6):393-403.

14. United Kingdom Prospective Diabetes Study (UKPDS) Group. Intensive blood-glucose control with sulphonylureas or insulin compared with conventional treatment risk of complications in patients with diabetes type 2 (UKPDS 33). [published erratum in Lancet 1999;354:602.]

15. Hughes VA, Frontera WR, Wood M, Evans WJ, Dallal GE Roubenoff R, et al. Longitudinal muscle strength changes in the elderly: influence of health, physical activity and body composition. J Gerontol A Biol Sci Med. 2001;56(5):B209-17.

16. Heymsfield SB, Wang J, Lichtman S, Kamen Y, Kehayias J, Pierson RN. Body composition in elderly subjects: A critical appraisal of clinical methodology. Am J Clin Nutr. 1989;50:1167-75.

17. Erberly LE, Cohen JD, Prineas R, Yang L. For the multiple risk factor intervention trial research group.Diabetes Care. 2003;26:848-54.

18. Haffner SM, Lehto S, Ronemaa T, Pyorala K, Laakso M. Mortality from coronary heart disease in subjects with type 2 diabetes and non diabetic subjects with and without prior myocardial infarction. N Engl J Med. 1998;339:229-34.

19. Thanopoulou AC, Karamanos BG, Angelico FC, AssaadKhalil AH, Barbato AF, Del Bem MP et al. Dietary fat intake as risk factor for the development of diabetes. Diabetes Care. 2003;26(2):302-7.

20. Panagiotakos DB, Zeimbekis A, Boutziouka V, Economou M, Kourlaba G, Toutouzas P, et al. Long-term fish intake is associated with better lipid profile, arterial blood pressure, and blood glucose levels in elderly people from Mediterranean islands (MEDIS epidemiological study). Med Sci Monit. 2007;13(7):CR307-12. 